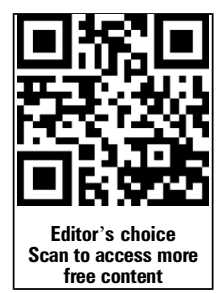

free content

\section{Handling editor Tore K Kvien \\ - Additional material is published online only. To view please visit the journal online (http://dx.doi.org/10.1136/ annrheumdis-2015-208263). \\ ${ }^{1}$ Clinical Epidemiology Unit, Department of Medicine Solna, Karolinska Institutet, Stockholm, Sweden \\ ${ }^{2}$ Department of Medical Epidemiology and Biostatistics, Karolinska Institutet, Stockholm, Sweden ${ }^{3}$ Rheumatology Unit, Department of Medicine Solna, Karolinska Institutet, Stockholm, Sweden}

\section{Correspondence to} Dr Hjalmar Wadström, Clinical Epidemiology Unit, Department of Medicine Solna, Karolinska Institutet, Stockholm, SE 171-76, Sweden; hjalmar.wadstrom@ki.se

Received 16 July 2015 Revised 1 November 2015 Accepted 14 December 2015 Published Online First 11 January 2016

\footnotetext{
To cite: Wadström $\mathrm{H}_{\text {, }}$ Frisell T, Sparén $P$, et al. Ann Rheum Dis

2016;75:1272-1278.
}

\title{
Do RA or TNF inhibitors increase the risk of cervical neoplasia or of recurrence of previous neoplasia? A nationwide study from Sweden
}

\author{
Hjalmar Wadström, ${ }^{1}$ Thomas Frisell, ${ }^{1}$ Pär Sparén, ${ }^{2}$ Johan Askling, ${ }^{1,3}$ on behalf of the \\ ARTIS study group
}

ABSTRACT

Objectives To examine screening patterns and the risk of cervical neoplasia in women with rheumatoid arthritis (RA) treated or not with tumour necrosis factor inhibitors (TNFi).

Methods We performed a nationwide register-based cohort study in Sweden of women with RA who started a first TNFi ( $n=9629)$, biologics-naive women with RA $(n=34984)$ and general population comparators (matched 1:10, $n=300$ 331), followed up from 1999 to 2012. Outcomes were first cytology screening with normal outcome, first ever cervical intraepithelial neoplasia (CIN) grade 1, first ever CIN 2-3 or adenocarcinoma in situ and first ever invasive cervical cancer during follow-up. HRs were assessed through Cox regressions adjusted for age, educational level, prior cervical screens, comorbidities, marital status and prior hospitalisations.

Results Biologic-naive women with RA had more screenings ( $\mathrm{HR} 1.08,95 \% \mathrm{Cl} 1.06$ to 1.10), were at greater risk of CIN 1 (HR 1.53, 1.23 to 1.89) and CIN 2-3 (HR 1.39, 1.16 to 1.66), but not of invasive cervical cancer (HR 1.09, 0.71 to 1.65) compared with the general population. Patients who initiated TNFi therapy had similar screening patterns (HR 1.01, 0.98 to 1.05), were not at increased risk of CIN 1 (HR 1.23, 0.87 to 1.74), but were at increased risk of CIN 2-3 (HR 1.36, 1.01 to 1.82 ) and invasive cervical cancer (HR 2.10, 1.04 to 4.23 ) compared with biologics-naive women with RA. Estimates varied little with successive adjustments, but were attenuated/absent in sensitivity analyses restricted to 2006-2012 and a diseasemodifying antirheumatic drugs-treated comparator. Conclusions Women with RA in general are at elevated risk of cervical dysplasia. Compared with biologics-naive patients, women treated with TNFi are at increased risk of cervical cancer. Whether this increase is causally linked with TNFi could not be fully disentangled.

\section{INTRODUCTION}

Data on the occurrence and progression of malignancies in patients with chronic systemic inflammatory diseases and with immunomodulatory treatments are limited. Immunosuppression is an established risk factor for virus-associated cancers including cervical cancer. ${ }^{1-3}$ Polymorphisms in the TNF gene have been associated with cervical cancer risk. ${ }^{4}$ Tumour necrosis factor inhibitors (TNFi), a cornerstone in the treatment of patients with rheumatoid arthritis (RA), are believed to decrease host defence against viral infections. ${ }^{5}$ Whether TNFi treatment increases the risk of virusassociated malignancies, such as cervical cancer, is not known.

Most guidelines suggest caution with TNFi treatment in patients with a history of cancer. Whether TNFi should be avoided in women with a history of premalignant cervical lesions is unknown. Two recent studies examined genital cancer risk among women with RA and a history of carcinoma in situ ${ }^{6}$ and any premalignant lesion of the cervix. ${ }^{7}$ In both, zero events were observed during follow-up of the TNFi groups, but the numbers of patients included $(\mathrm{n}=190 \text { and } 233)^{6}{ }^{7}$ were too small to rule out clinically meaningful risks.

Any increased risks among patients treated with TNFi should be interpreted in light of the risk associated with RA itself. A recent study found a $50 \%$ increased risk of high-grade dysplasia (cervical intraepithelial neoplasia (CIN) 2-3) or invasive cervical cancer among biologics-naive women with RA. ${ }^{8}$ Others have found decreased risks of invasive cervical cancer in $\mathrm{RA}^{9}{ }^{10}$ or no association. ${ }^{11}$ Cervical screening is an important determinant for cervical dysplasia and invasive malignancies, with a several-fold higher risk of cervical cancer in women not attending screening, already within $3-5$ years. $^{12}$

The aim of this study was to assess the incidence of premalignant and malignant cervical neoplasia in women with RA and in relation to TNFi treatment, taking cervical screening patterns and other risk factors into account.

\section{METHODS}

\section{Study design}

We performed a nationwide register-based cohort study, comparing the incidence of mild to severe cervical dysplasias CIN 1, CIN 2-3 (including cervical cancer in situ and adenocarcinoma in situ (AIS)), invasive cervical cancer and the use of cervical screening, across three large and populationbased cohorts: (a) biologics-naive women with RA starting a TNFi as the first ever biologic; (b) biologics-naive women with RA in general; and (c) the general Swedish female population.

\section{Setting}

The Swedish healthcare system is public and tax funded. Patients with RA are diagnosed and treated 
by rheumatologists. During the study period, TNFi was introduced in RA and use gradually increased to one-third of all patients.

\section{Data sources}

The registers used in this study have been described elsewhere. ${ }^{13}$ The Swedish biologics register (ARTIS) covers 90-95\% of all patients with RA treated with biologics in Sweden from 1999 to today. ${ }^{14} 15$ The Swedish Patient Register is a nationwide register with near complete coverage of all inpatient (since 1987) or non-primary outpatient visits (since 2001). Validations against medical files suggest that for many diagnoses (including RA), the validity is around $90 \% .^{16-18}$ This register was used to identify biologics-naive women with RA and comorbidities. The nationwide Prescribed Drug Register (data since 2005) was used to identify non-biological disease-modifying antirheumatic drug (DMARD) prescriptions. The Total Population Register was used to identify general population comparator subjects, date of birth and emigration or immigration. ${ }^{13}$ The Swedish Cancer Register has an estimated coverage of $>95 \%$ and was used to identify CIN 3 and invasive cervical cancer. ${ }^{19}$ The National Cervical Screening Program invites all women aged 23-60. The Swedish National Cervical Screening Registry gathers data on all Pap smears (and ensuing histology tests) in Sweden, both opportunistic and preplanned. More than $90 \%$ of all cervical cytology and histology tests are captured in the register. This register was used to identify screening visits 1995 or later, and histopathology for premalignant lesions (CIN 1-3 and AIS).

\section{Study population and follow-up}

Using ARTIS, we identified a cohort of patients with RA who initiated TNFi as their first ever biologic. Start of follow-up was defined as date of TNFi start.

Using the Patient Register, we identified all women with two or more visits in non-primary outpatient care listing a main or contributory diagnosis of RA. At least one of these had to be at a rheumatology or internal medicine department. We excluded patients who had ever received a diagnosis of juvenile idiopathic arthritis, ankylosing spondylitis, systemic lupus erythematosus or psoriatic arthritis. Through linkage to ARTIS, we could thus define a cohort of patients with RA who were biologics-naive (and followed prospectively until first biologics initiation). Patients who started a TNFi treatment during follow-up thereafter contributed to the TNFi cohort. Start of follow-up was defined as the first date when all the inclusion criteria were fulfilled 2001-2012.

Through linkage of the biologics-naive cohort to the Population Register, we matched (1:10, by year of birth, sex, county of residence and vital status at the date of first identification of RA) a general population comparator cohort. The inclusion date was set to that of their corresponding biologics-naive patient with RA.

End of follow-up was defined as first of 31 December 2012, death, last emigration, a total hysterectomy, date of any solid organ transplantation and occurrence of the outcome under study.

\section{Outcomes}

Considering the exploratory nature of our study, we assessed the following outcomes in parallel:

1. First cytology screening (planned or opportunistic) with a normal outcome during follow-up.

2. First CIN 1 (including atypical glandular cells) during follow-up in individuals without any history of cervical dysplasia or invasive cervical cancer before (excluded) or during (censored) follow-up.

3. First CIN 2-3 or AIS (CIN 2+) during follow-up in individuals without any history of CIN 2+ at start of follow-up or invasive cervical cancer before or during follow-up, but irrespective of CIN 1.

4. First invasive cervical cancer during follow-up in individuals without a history of invasive cervical cancer at start of follow-up, but irrespective of any cervical dysplasia before or during follow-up. We also assessed the risks for invasive cervical cancer among patients with a history of cervical dysplasia ever, a history of cervical dysplasia during the five years before start of follow-up and a history of a normal screening test (and no subsequent pathological screening tests) during the five years before start of follow-up.

\section{Covariates and statistical analyses}

We quantified the cumulative number of Pap smears and time since the most recent Pap smear, during the five years prior to start of follow-up, whether as preplanned or opportunistic screening, and also identified any occurrence of cervical dysplasia or invasive cervical cancer during the same time period. Data on marital status and highest achieved education at the index date were retrieved from Statistics Sweden.

Through linkage to the Swedish Patient Register, we identified hospitalisation or outpatient visits listing ischaemic heart disease, chronic obstructive pulmonary disease, diabetes mellitus and knee/ankle/hip/shoulder-joint surgery as proxies for intensity of healthcare contacts. These comorbidities were treated as separate time-varying covariates and were allowed to change (from absent to present, but not back) up until 365 days before end of follow-up. Overall healthcare utilisation was measured as total days spent in hospital during the five years prior to start of follow-up. The effect of previous invasive cancer other than cervical cancer was assessed in a sensitivity analysis. Information on parity and family history was only available for a study subset through 2010.

We calculated the total number of events, person-years at risk and incidence rate of each outcome in each cohort. We used Cox regression (where the smallest cell contained five or more events) with age as timescale to estimate HRs. In the first Cox model, we stratified by decade of birth, in the second we further adjusted for educational level, marital status and number of Pap smears before start of follow-up, and in the full model we further added the above-mentioned comorbidities. Alternative timescales (here, follow-up time and calendar time) and the three model specifications yielded similar results. The main analysis was stratified by time since start of follow-up. To investigate any non-proportionality of HRs, we plotted hazard functions, stratified the analyses on attained age (three age bands) and estimated crude as well as age-standardised incidences of each outcome in each cohort. SAS V.9.4 was used.

\section{Sensitivity analyses}

To assess more contemporary biologics-initiators and to reduce the potential for channelling, we restricted the study period to 1 January 2006 through 31 December 2012 and the biologicsnaive RA cohort to patients who (in addition to fulfilling the entry criteria to this cohort) initiated or were on treatment (concomitantly or successively) with at least two non-biological DMARDs.

To assess the impact of risk window definition, follow-up for the TNFi cohort was stopped 90 days after discontinuation of the biologic in question ('on drug'), with or without attention 
to the individual TNFi drug ('on class') instead of 'ever-since first exposure'.

\section{RESULTS}

The main analysis included 9629 TNFi initiators, 34984 biologics-naive women with RA and 300331 general population comparators. The TNFi cohort was younger (56 years), had higher educational level and had a burden of comorbidities that was intermediate to the rates in the general population and the biologics-naive cohort (table 1). In the subset below 60 years of age, the screening intensity was similar across all cohorts.

\section{Occurrence and relative risk of cervical malignancies: biologics-naive RA versus the general population}

The crude incidence of a first screening with a normal outcome during follow-up was similar between the biologics-naive RA cohort and the general population cohort, although the fully adjusted HR was statistically significantly increased (HR 1.08, 95\% CI 1.06 to 1.10 ). There were statistically significant higher rates in the biologics-naive RA cohort of CIN 1 (HR 1.53, 95\% CI 1.23 to 1.89 ) and CIN 2+ (HR 1.39, 95\% CI 1.16 to 1.66 ) but not of invasive cancer (HR 1.09 , 95\% CI 0.71 to 1.65 ) (table 2). When we restricted the analyses to individuals with a history of CIN 1 or CIN $2+$ ever and during the five years before start of follow-up, we observed four and two cases of invasive cervical cancer, respectively, in the RA population. When restricting to individuals with a history of a normal screening test before start of follow-up, we observed seven cases of invasive cervical cancer, corresponding to an HR of 0.78 (95\% CI 0.36 to 1.70 ) (table 2).

\section{Occurrence and relative risk of cervical malignancies: TNFi initiators versus biologics-naive RA}

In the TNFi cohort, the crude but not the adjusted incidence of a first screening with a normal outcome was higher than in the biologics-naive RA cohort (HR 1.01, 95\% CI 0.98 to 1.05). There was no statistically significant difference in risk for CIN 1 (HR 1.23, 95\% CI 0.87 to 1.74 ), a higher rate of CIN 2+ (HR $1.36,95 \%$ CI 1.01 to 1.82 ) and a doubled risk of invasive cervical cancer in the TNFi cohort (HR 2.10, 95\% CI 1.04 to 4.23) (table 2). When we restricted the analyses to individuals with a history of CIN 1 or CIN $2+$ ever, and during the five years before start of follow-up, we observed three and one case (s) of invasive cervical cancer, respectively. When restricting to

Table 1 Characteristics of the study population of Swedish women with rheumatoid arthritis (RA) (biologics-naive and starting a first ever tumour necrosis factor inhibitor (TNFi) treatment 1999-2012, respectively), and matched general population comparator subjects

\begin{tabular}{|c|c|c|c|}
\hline $\begin{array}{l}\text { Characteristics at start of follow-up } \\
n\end{array}$ & $\begin{array}{l}\text { TNFi initiators } \\
9629\end{array}$ & $\begin{array}{l}\text { Biologics-naive RA } \\
34984\end{array}$ & $\begin{array}{l}\text { General female population } \\
300331\end{array}$ \\
\hline Age at entry (median, min-max) & $56(18-92)$ & $62(18-97)$ & $61(18-96)$ \\
\hline Year of birth (median, min-max) & 1949 (1907-1994) & 1943 (1906-1994) & $1944(1906-1994)$ \\
\hline Year of entry (median, min-max) & $2006(1999-2012)$ & $2004(2001-2012)$ & $2004(2001-2012)$ \\
\hline Time since RA onset (years) & 8.2 & NA & NA \\
\hline DAS28 (median, IQR) at biologics start & $5.30(4.40-6.13)$ & NA & NA \\
\hline HAQ (median, IQR) at biologics start & $1.25(0.88-1.75)$ & NA & NA \\
\hline Concomitant DMARDs (\%) & 71 & NA & NA \\
\hline Concomitant oral steroids (\%) & 47 & NA & NA \\
\hline \multicolumn{4}{|l|}{ Educational level, years (\%) } \\
\hline$\leq 9$ & 25 & 36 & 33 \\
\hline $9-12$ & 44 & 40 & 40 \\
\hline$>12$ & 31 & 22 & 26 \\
\hline \multicolumn{4}{|l|}{ Comorbidities (\%) } \\
\hline Chronic obstructive pulmonary disease & 2.4 & 3.3 & 1.9 \\
\hline Diabetes mellitus & 5.2 & 5.8 & 4.2 \\
\hline Ischaemic heart disease & 4.2 & 7.8 & 5.7 \\
\hline Previous joint surgery & 27.8 & 20.4 & 5.2 \\
\hline Total $\mathrm{n}$ days spent in hospital during last five years, median (IQR) & $1(0-10)$ & $1(0-9)$ & $0(0-2)$ \\
\hline \multicolumn{4}{|l|}{ Cervical screening characteristics, all individuals } \\
\hline Mean $\mathrm{n}$ cervical screens during last five years preceding start of follow-up (range) & $1.17(0-12)$ & $0.89(0-15)$ & $0.91(0-19)$ \\
\hline Median time since last cervical screening visit at date of start of follow-up (days, range) & $825(1-6198)$ & $937(1-7718)$ & $895(1-16626)$ \\
\hline CIN 1 within five years before start of FU (\%) & 0.5 & 0.4 & 0.3 \\
\hline CIN 2+ within five years before start of FU (\%) & 0.7 & 0.5 & 0.4 \\
\hline Mean number of cervical screens during follow-up, (range) & $1.08(0-19)$ & $0.65(0-18)$ & $0.87(0-24)$ \\
\hline \multicolumn{4}{|l|}{ Cervical screening characteristics, individuals below 60 years of age at baseline } \\
\hline Mean $\mathrm{n}$ cervical screens during last five years preceding start of follow-up (range) & $1.44(0-12)$ & $1.39(0-15)$ & $1.39(0-19)$ \\
\hline Median time since last cervical screening visit at date of start of follow-up (days, range) & $655(1-6198)$ & $686(1-6476)$ & $676(1-6520)$ \\
\hline CIN 1 within five years before start of follow-up (\%) & 0.7 & 0.7 & 0.5 \\
\hline CIN $2+$ within five years before start of follow-up (\%) & 1.0 & 1.0 & 0.8 \\
\hline Mean number of cervical screens during follow-up, (range) & $1.56(0-19)$ & $1.23(0-18)$ & $1.60(0-24)$ \\
\hline
\end{tabular}

CIN, cervical intraepithelial neoplasia; DAS28, disease activity score 28; DMARD, disease-modifying antirheumatic drug; FU, follow-up; HAQ, health assessment questionnaire; NA, not available. 


\begin{tabular}{|c|c|c|c|c|c|c|c|}
\hline $\begin{array}{l}\text { Outcome } \\
\text { definition }\end{array}$ & Cohort & $\begin{array}{l}\text { Number of } \\
\text { patients at risk }\end{array}$ & $\begin{array}{l}\text { Mean } \\
\text { follow-up }\end{array}$ & $\begin{array}{l}\text { Number } \\
\text { of events }\end{array}$ & $\begin{array}{l}\text { Crude incidence per } \\
100000 \text { person-years }\end{array}$ & $\mathrm{HR}^{*}$ & $\mathrm{HR}^{*}$ \\
\hline \multirow{3}{*}{$\begin{array}{l}\text { First screening } \\
\text { with normal result }\end{array}$} & TNFi & 9072 & 3.29 & 4362 & 14599 & 1.01 (0.98 to 1.05$)$ & \\
\hline & Biologics-naive RA & 33253 & 3.57 & 10958 & 9224 & Ref & 1.08 (1.06 to 1.10$)$ \\
\hline & General population & 287602 & 4.18 & 114943 & 9556 & & Ref \\
\hline \multirow[t]{3}{*}{ CIN 1} & TNFi & 8943 & 6.10 & 52 & 95.3 & 1.23 (0.87 to 1.74$)$ & \\
\hline & Biologics-naive RA & 32947 & 5.26 & 99 & 57.1 & Ref & 1.53 (1.23 to 1.89$)$ \\
\hline & General population & 285232 & 6.66 & 852 & 44.8 & & Ref \\
\hline \multirow[t]{3}{*}{ CIN $2+$} & TNFi & 9018 & 6.11 & 75 & 136.0 & 1.36 (1.01 to 1.82$)$ & \\
\hline & Biologics-naive RA & 33130 & 5.27 & 137 & 78.5 & Ref & 1.39 (1.16 to 1.66$)$ \\
\hline & General population & 286731 & 6.67 & 1332 & 69.7 & & Ref \\
\hline \multirow{3}{*}{$\begin{array}{l}\text { Invasive cervical } \\
\text { cancer, overall }\end{array}$} & TNFi & 9629 & 6.15 & 14 & 23.7 & 2.10 (1.04 to 4.23$)$ & \\
\hline & Biologics-naive RA & 34984 & 5.27 & 25 & 13.6 & Ref & 1.09 (0.71 to 1.65$)$ \\
\hline & General population & 300331 & 6.68 & 275 & 13.7 & & Ref \\
\hline \multirow{3}{*}{$\begin{array}{l}\text { Invasive cervical } \\
\text { cancert }\end{array}$} & TNFi & 107 & 6.76 & 1 & 138.2 & $\ddagger$ & \\
\hline & Biologics-naive RA & 260 & 5.47 & 2 & 140.6 & Ref & $\ddagger$ \\
\hline & General population & 2119 & 6.72 & 5 & 35.1 & & Ref \\
\hline \multirow{3}{*}{$\begin{array}{l}\text { Invasive cervical } \\
\text { cancer§ }\end{array}$} & TNFi & 685 & 6.05 & 3 & 72.3 & $\ddagger$ & \\
\hline & Biologics-naive RA & 2036 & 5.06 & 4 & 38.8 & Ref & $\ddagger$ \\
\hline & General population & 15069 & 6.47 & 20 & 20.5 & & Ref \\
\hline \multirow{3}{*}{$\begin{array}{l}\text { Invasive cervical } \\
\text { cancerql }\end{array}$} & TNFi & 6418 & 6.50 & 10 & 24.0 & 3.77 (1.35 to 10.48$)$ & \\
\hline & Biologics-naive RA & 18520 & 5.47 & 7 & 6.9 & Ref & 0.78 (0.36 to 1.70$)$ \\
\hline & General population & 162543 & 7.08 & 112 & 9.7 & & Ref \\
\hline
\end{tabular}

*Stratified on decade of birth and adjusted for educational level, number of cervical screens past five years, comorbidities, marital status and total days spent in hospital during last five years, also implicitly adjusted for age since age was used as the model's timescale.

tIn individuals with a history of CIN 1 or CIN 2+ during the five years before start of follow-up.

$\ddagger$ Not computed due to low number of events.

§in individuals with a history of CIN 1 or CIN 2+ ever.

IIn individuals with a normal screening test as the most recent screening test less than five years before start of follow-up.

CIN, cervical intraepithelial neoplasia; RA, rheumatoid arthritis; TNFi, tumour necrosis factor inhibitor.

individuals with a normal screening test as the last screening before start of follow-up, we observed 10 cases of invasive cervical cancer, corresponding to an HR of 3.77 (95\% CI 1.35 to 10.48) (table 2).

\section{Time since start of treatment}

For CIN 1 and CIN 2+ and comparing TNFi initiators versus biologics-naive RA, there were no obvious differences in HR across follow-up (if anything a downward trend in HRs) (table 3). For invasive cervical cancer, small numbers limited comparisons.

\section{Stage at diagnosis of invasive cervical cancer}

There were no obvious differences in stage at diagnosis of invasive cervical cancer according to the International Federation of Gynecology and Obstetrics staging system, ${ }^{20}$ although numbers were small and missingness was not negligible though equal across cohorts (see online supplementary table $\mathrm{S} 4$ ).

\section{Sensitivity analyses}

When the study period was restricted to 2006-2012 and the biologics-naive comparator restricted to those individuals who had received dispensing of two or more different csDMARDs, the association with TNFi (defined as incident or ongoing 2006 or later) and invasive cervical cancer was attenuated (HR 1.36, 95\% CI 0.59 to 3.13) and absent when restricted to new users of TNFi 2006 or later (one case during 18110 person-years). Further sensitivity analyses of the full cohort and study period but with risk windows defined as 'on drug' (HR 1.46, 95\% CI 0.54 to 3.94 ) and 'on class' (HR $1.81,95 \%$ CI 0.78 to 4.18 ) attenuated the strength of the observed association between TNFi initiation and risk of invasive cervical cancer. All other sensitivity analyses provided results similar to the main analysis (table 4 and online supplementary tables S1-S3).

\section{DISCUSSION}

We noted a modest increase in risk of CIN 1 and CIN $2+$ but not of invasive cervical cancer in biologics-naive women with RA versus the general population, a finding that was not readily explained by differences in cervical screening. Among women with RA initiating TNFi therapy, the screening patterns were largely similar to those of other women with RA, the risks for CIN 1 and CIN 2+ were 20-40\% increased and the risk for invasive cervical cancer was doubled, although with wide confidence limits.

A previous study by Kim et $a l^{21}$ reported similar cervical screening attendance in RA and the general population. Most other studies have demonstrated suboptimal screening attendance among women with chronic diseases, including RA. ${ }^{22-24}$ The finding in our study that RA might be, but is not strongly, linked with CIN 3/invasive cervical cancer is in line with most, ${ }^{8} 1125$ but not all, ${ }^{910}$ previous studies. The three available studies on TNFi and aspects of cervical malignancy have not demonstrated an increased risk with TNFi. ${ }^{6}{ }^{26}$ Two of them only included women with a history of cervical neoplasia. The third study did not condition on a history of cervical neoplasia but was smaller than this study (about 8000 person-years vs 60000 person-years in our TNFi cohort) ${ }^{26}$ and did not accommodate, for example, cervical screening. 
Table 3 HRs and 95\% Cls, number of persons, events and person-years, comparing tumour necrosis factor inhibitor (TNFi) versus biologics-naive rheumatoid arthritis (RA) for each outcome

\begin{tabular}{|c|c|c|c|c|}
\hline Outcome & $0-2$ years & $2-5$ years & $5+$ years & Overall \\
\hline \multicolumn{5}{|l|}{ CIN 1} \\
\hline$H R^{*}$ & 1.77 (1.14 to 2.73$)$ & 1.05 (0.61 to 1.79$)$ & $0.74(0.37$ to 1.49$)$ & 1.23 (0.87 to 1.74$)$ \\
\hline $\mathrm{N}$ events, TNFi & 27 & 16 & 9 & 52 \\
\hline $\mathrm{N}$ patients, TNFi & 8943 & 7418 & 5007 & 8943 \\
\hline Pyr, TNFi & 16474 & 18609 & 19499 & 54581 \\
\hline \multicolumn{5}{|l|}{ CIN 2+ } \\
\hline $\mathrm{HR}^{*}$ & 1.55 (1.04 to 2.32$)$ & $1.39(0.92$ to 2.10$)$ & $1.06(0.63$ to 1.78$)$ & $1.36(1.01$ to 1.82$)$ \\
\hline $\mathrm{N}$ events, TNFi & 30 & 28 & 17 & 75 \\
\hline N patients, TNFi & 9018 & 7493 & 5066 & 9018 \\
\hline Pyr, TNFi & 16629 & 18808 & 19707 & 55144 \\
\hline \multicolumn{5}{|c|}{ Invasive cervical cancer } \\
\hline $\mathrm{HR}^{*}$ & $\dagger$ & $\dagger$ & 3.22 (1.37 to 7.58$)$ & $2.10(1.04$ to 4.23$)$ \\
\hline $\mathrm{N}$ events, TNFi & 4 & 2 & 8 & 14 \\
\hline $\mathrm{N}$ patients, TNFi & 9629 & 8021 & 5449 & 9629 \\
\hline Pyr, TNFi & 17774 & 20166 & 21251 & 59191 \\
\hline
\end{tabular}

Stratified by time since start of follow-up ( $<2$ years, $2-5$ years, $5+$ years).

${ }^{*}$ Stratified on decade of birth and adjusted for educational level, number of cervical screens past five years, comorbidities, marital status and total days spent in hospital during last five years, also implicitly adjusted for age since age was used as the model's timescale.

tNot computed due to low number of events.

$\mathrm{CIN}$, cervical intraepithelial neoplasia.

Table 4 Sensitivity analyses restricted to 2006-2012, on first tumour necrosis factor inhibitor (TNFi) ('on drug') and continuous time on TNFi treatment ("on class'), respectively

\begin{tabular}{|c|c|c|c|c|c|}
\hline Analysis & Outcome & Cohort comparison & $\mathrm{n}$ events & Person-years & $H^{*}$ * \\
\hline \multirow{12}{*}{$\begin{array}{l}2006-2012 \text { and stricter definition } \\
\text { of biologics-naive RAt }\end{array}$} & \multirow[t]{4}{*}{ CIN 1} & TNFi & 40 & 41938 & 1.02 (0.67 to 1.54$)$ \\
\hline & & New TNFi usersł & 21 & 16725 & $1.12(0.67$ to 1.87$)$ \\
\hline & & Biologics-naive RA & 59 & 88696 & Ref \\
\hline & & General population & 637 & 1394519 & 0.59 (0.45 to 0.78$)$ \\
\hline & \multirow[t]{4}{*}{$\mathrm{CIN} 2+$} & TNFi & 58 & 42429 & 1.10 (0.72 to 1.68$)$ \\
\hline & & New TNFi usersł & 30 & 16916 & $1.09(0.72$ to 1.66$)$ \\
\hline & & Biologics-naive RA & 89 & 89385 & Ref \\
\hline & & General population & 1003 & 1403802 & 0.59 (0.47 to 0.73$)$ \\
\hline & \multirow[t]{4}{*}{ Invasive cervical cancer } & TNFi & 10 & 45557 & 1.36 (0.59 to 3.13$)$ \\
\hline & & New TNFi users $\ddagger$ & 1 & 18110 & $\S$ \\
\hline & & Biologics-naive RA & 17 & 94590 & Ref \\
\hline & & General population & 207 & 1474695 & $0.73(0.44$ to 1.21$)$ \\
\hline \multirow[t]{6}{*}{ On drug } & \multirow[t]{2}{*}{ CIN 1} & TNFi & 30 & 29094 & 1.23 (0.81 to 1.88$)$ \\
\hline & & Biologics-naive RA & 99 & 173369 & Ref \\
\hline & \multirow[t]{2}{*}{ CIN 2+ } & TNFi & 50 & 29407 & 1.60 (1.15 to 2.22$)$ \\
\hline & & Biologics-naive RA & 137 & 174514 & Ref \\
\hline & \multirow[t]{2}{*}{ Invasive cervical cancer } & TNFi & 5 & 31499 & 1.46 (0.54 to 3.94$)$ \\
\hline & & Biologics-naive RA & 25 & 184391 & Ref \\
\hline \multirow[t]{6}{*}{ On class } & \multirow[t]{2}{*}{ CIN 1} & TNFi & 37 & 36699 & 1.21 (0.82 to 1.78$)$ \\
\hline & & Biologics-naive RA & 99 & 173369 & Ref \\
\hline & \multirow[t]{2}{*}{ CIN 2+ } & TNFi & 59 & 37100 & 1.50 (1.09 to 2.05$)$ \\
\hline & & Biologics-naive RA & 137 & 174514 & Ref \\
\hline & \multirow[t]{2}{*}{ Invasive cervical cancer } & TNFi & 8 & 39845 & 1.81 (0.78 to 4.18$)$ \\
\hline & & Biologics-naive RA & 25 & 184391 & Ref \\
\hline
\end{tabular}

HRs (HRs model a-c) and 95\% Cls comparing each outcome between cohorts.

* Stratified on decade of birth and adjusted for educational level, number of cervical screens past five years, comorbidities, marital status and total days spent in hospital during last five years, also implicitly adjusted for age since age was used as the model's timescale.

TThe biologics-naive RA cohort was restricted to patients who initiated or were on treatment with at least two of any of the commonly used non-biological DMARDs (methotrexate, sulfasalazine, antimalarials or leflunomide).

¥TNFi cohort restricted to patients who started their first TNFi treatment 2006 or later.

$\S$ Not computed due to low number of events.

CIN, cervical intraepithelial neoplasia; DMARD, disease-modifying antirheumatic drug; RA, rheumatoid arthritis. 
Assessments of the role of specific antirheumatic treatments on the risk of cervical cancer pose methodological challenges including confounding and channelling bias, detection/lead time bias and statistical precision, each of which may have a bearing on the interpretation of our results: unlike many other cancer types, the age-incidence curve for cervical cancer in screened populations is almost flat from age $30 .{ }^{12}$ Further, the 'risk' of having a malignancy detected through screening decreases as the screening programme ends at 60 years of age. In our study, there were age differences between the cohorts, the mean age of which was around 60 years of age, such that a crude comparison of baseline characteristics and crude incidences would be biased. Therefore, age was used as timescale in our Cox regressions, and sensitivity analyses restricted to ages 18-60 were performed.

Invasive cervical cancer arises from human papillomavirus (HPV) infection via mild to severe dysplasia to invasive malignancies, ${ }^{27}$ providing the rationale for screening aimed at detecting premalignant lesions and reducing longer-term risks of invasive cancer. Since almost all observed premalignant lesions are detected through screening, screening intensity is a strong driver of the reported incidence of cervical premalignancies. Conversely, screening non-attendance is a strong risk factor for the development of invasive cervical cancer. ${ }^{12}{ }^{28}$ Although we adjusted for screening attendance before start of follow-up, and although there were no marked differences in screening attendance between the cohorts, there might be residual confounding or qualitative differences in the number, timing, reasons for and outcome of the screening tests delivered. Conversely, analyses restricted to individuals with a normal screening visit during the five years preceding follow-up fell close to the main analyses, and adjustment for screening as a time-fixed binary or timevarying binary covariate did not markedly alter the results.

While we could adjust for several potential confounders, our analyses could not fully accommodate any differences in the accumulated burden of RA disease or the accumulated exposure to antirheumatic drugs other than TNFi/biologics. Patients in our study whose RA was severe enough to warrant TNFi treatment probably have a higher lifetime exposure to DMARDs and cytotoxic agents. This in turn may be linked with an increased susceptibility to virus-associated malignancies. For this reason, unmeasured and residual confounding as well as channelling bias cannot be excluded as explanations for the observed differences between the TNFi cohort and the biologics-naive RA comparator, which were not seen when the study period was restricted to 2006 or later and treatment criteria were added to the RA comparator. Unfortunately, we could not adjust for HPV/sexually transmitted diseases or smoking. ${ }^{29}{ }^{30}$ However, differences in prevalence of smoking between biologics-naive and TNFi-treated patients with RA in Sweden are small. ${ }^{31}$ Even though we were able to use nationwide registers with high coverage as a source for data on screening attendance and cervical (pre-)malignancies, small numbers limited comparisons across different strata, models and sensitivity analyses.

Despite the inherent methodological challenges, our study has several strengths: it is based on national registers with almost complete coverage, ensuring generalisability. Although statistical precision was sometimes limited, our study is among the largest to date. Through linkage to national registers and the cervical screening register, we had the possibility to assess and adjust for several important potential confounders. Besides cervical screening, we could adjust for level of education, which is a risk factor for developing RA, for starting a biologic, for screening nonattendance and for cervical cancer. We could also adjust for comorbidities and number of healthcare contacts, serving as markers of frailty and proxies for accumulated RA disease severity, which was also associated with a lower use of screening services. We could demonstrate that parity and family history of malignancies were probably not important confounders.

To conclude, our study suggests that women with RA in general are at elevated risk of cervical dysplasia and that women with RA who initiate TNFi treatment may be at increased risk of cervical cancer, though the extent to which this is reflective of bias or causality could not be fully disentangled. Whether causally linked or not, translated to the clinical setting, our finding of a $50-100 \%$ increase in the risk of invasive cervical cancer in women initiating TNFi therapy is weaker than, for example, the risk associated with screening non-attendance (around 100$200 \%$ increase in risk $)^{28}$ and would correspond to one additional annual case for every 7000-15000 treated women, which needs to be weighed against any benefit of TNFi treatment or its alternatives.

Acknowledgements The ARTIS Study Group conducts scientific analyses using data from the Swedish Biologics Register ARTIS run by the Swedish Society for Rheumatology.

Funding For work with this register, the Swedish Society for Rheumatology has received funding, independent of the exact conduct of these scientific analyses, from Abbott/Abbvie, Bristol-Myers Squibb, Merck \& Co. Inc., Sobi, AstraZeneca, Pfizer, $U C B$ and Roche. This study also received funding through an agreement with Merck \& Co. Inc., in response to a regulatory request, and by grants from The Swedish Foundation for Strategic Research, the Swedish Cancer Society, ALF and the Swedish Research Council.

Collaborators The following were members of the ARTIS Study Group at the time of study initiation: Johan Askling, Lars Klareskog, Staffan Lindblad, Sofia Ernestam, Nils Feltelius and Ronald van Vollenhoven (Karolinska Institutet, Stockholm, Sweden), Eva Baecklund (Uppsala University, Uppsala, Sweden), Alf Kastbom (Linköping University, Linköping, Sweden), Helena Forsblad and Lennart Jacobsson (Sahlgrenska Academy, Göteborg, Sweden), Pierre Geborek and Lars-Erik Kristensen (Lund University, Malmö/Lund, Sweden) and Solbritt Rantapää-Dahlqvist (Umeå University, Umeå, Sweden)

Contributors HW had full access to all of the data in the study and takes responsibility for the integrity of the data and the accuracy of the data analysis. HW conducted the statistical analyses. All authors participated in designing the analyses, interpreting the results and writing the manuscript.

Competing interests TF has served on an advisory board for Pfizer. JA has/has had research grants with AstraZeneca, Pfizer, BMS, UCB, Abbvie, Roche and Merck.

Ethics approval The study was approved by the ethics committee at Karolinska Institutet.

Provenance and peer review Not commissioned; externally peer reviewed.

\section{REFERENCES}

1 Vajdic CM, McDonald SP, McCredie MR, et al. Cancer incidence before and after kidney transplantation. JAMA 2006;296:2823-31.

2 Krynitz B, Edgren $\mathrm{G}$, Lindelof $\mathrm{B}$, et al. Risk of skin cancer and other malignancies in kidney, liver, heart and lung transplant recipients 1970 to 2008-a Swedish population-based study. Int I Cancer 2013;132:1429-38.

3 Grulich AE, van Leeuwen MT, Falster MO, et al. Incidence of cancers in people with HIVIAIDS compared with immunosuppressed transplant recipients: a meta-analysis. Lancet 2007;370:59-67.

4 Jin Y. Association of single nucleotide polymorphisms in tumor necrosis factor-alpha with cervical cancer susceptibility. Cell Biochem Biophys 2015;71:77-84.

5 Che H, Lukas C, Morel J, et al. Risk of herpes/herpes zoster during anti-tumor necrosis factor therapy in patients with rheumatoid arthritis. Systematic review and meta-analysis. Joint Bone Spine 2014;81:215-21.

6 Mercer LK, Low AS, Galloway JB, et al. Anti-TNF therapy in women with rheumatoid arthritis with a history of carcinoma in situ of the cervix. Ann Rheum Dis 2013;72:143-4.

7 Cordtz R, Mellemkjær L, Glintborg B, et al. Malignant progression of precancerous lesions of the uterine cervix following biological DMARD therapy in patients with arthritis. Ann Rheum Dis 2015;74:1479-80.

8 Kim SC, Glynn RJ, Giovannucci E, et al. Risk of high-grade cervical dysplasia and cervical cancer in women with systemic inflammatory diseases: a population-based cohort study. Ann Rheum Dis 2015;74:1360-7. 
9 Parikh-Patel $\mathrm{A}$, White $\mathrm{RH}$, Allen $\mathrm{M}$, et al. Risk of cancer among rheumatoid arthritis patients in California. Cancer Causes Control 2009;20:1001-10.

10 Chen YJ, Chang YT, Wang CB, et al. The risk of cancer in patients with rheumatoid arthritis: a nationwide cohort study in Taiwan. Arthritis Rheum 2011;63:352-8.

11 Mellemkjaer L, Linet MS, Gridley G, et al. Rheumatoid arthritis and cancer risk. Eur J Cancer 1996:32A:1753-7.

12 Azerkan F, Sparén P, Sandin S, et al. Cervical screening participation and risk among Swedish-born and immigrant women in Sweden. Int I Cancer 2012;130:937-47.

13 Askling J, Fored CM, Geborek $\mathrm{P}$, et al. Swedish registers to examine drug safety and clinical issues in RA. Ann Rheum Dis 2006:65:707-12.

14 Neovius M, Simard J, Sundström A, et al. Generalisability of clinical registers used for drug safety and comparative effectiveness research: coverage of the Swedish Biologics Register. Ann Rheum Dis 2011;70:516-19.

15 Wadström H, Eriksson J, Neovius M, et al., ARTIS Study Group. How good is the coverage and how accurate are exposure data in the Swedish Biologics Register (ARTIS)? Scand I Rheumatol 2015:44:22-8.

16 Waldenlind K, Eriksson J, Grewin B, et al. Validation of the rheumatoid arthritis diagnosis in the Swedish National patient register: a cohort study from Stockholm County. BMC Musculoskelet Disord 2014;15:432.

17 Knight A, Sandin S, Askling J. Increased risk of autoimmune disease in families with Wegener's Granulomatosis. J Rheumatol 2010;37:2553-8.

18 Baecklund E, Ekbom A, Sparén $P$, et al. Disease activity and risk of lymphoma in patients with rheumatoid arthritis: nested case-control study. BMJ 1998;317: 180-1.

19 Barlow L, Westergren K, Holmberg L, et al. The completeness of the Swedish Cancer Register-a sample survey for year 1998. Acta Oncol 2009;48:27-33.

20 Pecorelli S, Benedet JL, Creasman WT, et al. FIGO staging of gynecologic cancer 1994-1997 FIGO Committee on Gynecologic Oncology. International Federation of Gynecology and Obstetrics. Int J Gynecol Obstet 1999;64:5-10.
21 Kim SC, Schneeweiss S, Myers JA, et al. No differences in cancer screening rates in patients with rheumatoid arthritis compared to the general population. Arthritis Rheum 2012;64:3076-82.

22 Kremers HM, Bidaut-Russell M, Scott CG, et al. Preventive medical services among patients with rheumatoid arthritis. J Rheumatoly 2003;30:1940-7.

23 Kiefe $C$, Funkhouser $E_{\text {, Fouad } M}$, et al. Chronic disease as a barrier to breast and cervical cancer screening. J Gen Intern Med 1998;13:357-65.

24 MacLean $\mathrm{CH}$, Louie $\mathrm{R}$, Leake $\mathrm{B}$, et al. QUality of care for patients with rheumatoid arthritis. JAMA 2000;284:984-92.

25 Gridley G, McLaughlin JK, Ekbom A, et al. Incidence of cancer among patients with rheumatoid arthritis. J Natl Cancer Inst 1993:85:307-11.

26 Carmona L, Abasolo L, Descalzo MA, et al. Cancer in patients with rheumatic diseases exposed to TNF antagonists. Semin Arthritis Rheum 2011:41:71-80.

27 Woodman CBJ, Collins SI, Young LS. The natural history of cervical HPV infection: unresolved issues. Nat Rev Cancer 2007;7:11-22.

28 Andrae B, Kemetli L, Sparén $\mathrm{P}$, et al. Screening-preventable cervical cancer risks: evidence from a nationwide audit in Sweden. J Natl Cancer Inst 2008:100:622-9.

29 Appleby P, Beral V, Berrington de González A, et al., International Collaboration of Epidemiological Studies of Cervical Cancer. Carcinoma of the cervix and tobacco smoking: collaborative reanalysis of individual data on 13,541 women with carcinoma of the cervix and 23,017 women without carcinoma of the cervix from 23 epidemiological studies. Int J Cancer 2006;118:1481-95.

30 Kjellberg L, Hallmans G, Ahren AM, et al. Smoking, diet, pregnancy and oral contraceptive use as risk factors for cervical intra-epithelial neoplasia in relation to human papillomavirus infection. Br J Cancer 2000;82:1332-8.

31 Saevarsdottir $S$, Wedrén $S$, Seddighzadeh $M$, et al. Patients with early rheumatoid arthritis who smoke are less likely to respond to treatment with methotrexate and tumor necrosis factor inhibitors: Observations from the Epidemiological Investigation of Rheumatoid Arthritis and the Swedish Rheumatology Register cohorts. Arthritis Rheum 2011:63:26-36. 